\title{
A Correlation Analysis Model of Human Factors in Mine Accidents Based on Apriori Algorithm
}

\author{
Yunfeng Song ${ }^{1,2}$ \\ ${ }^{1}$ School of Management, China University of Mining and Technology-Beijing, Beijing 100083, China \\ ${ }^{2}$ School of Management Engineering and Business, Hebei University of Engineering, Handan 056038, China
}

Corresponding Author Email: tbp1600501009@student.cumtb.edu.cn

https://doi.org/10.18280/ijsse.100314

Received: 19 January 2020

Accepted: 5 May 2020

\section{Keywords:}

human factors, mine accidents, correlation analysis, human reliability, Apriori algorithm, neural network (NN), principal component analysis (PCA)

\begin{abstract}
Human factors are the main cause of mine accidents. With the aid of the Apriori algorithm, this paper establishes a new correlation analysis model for human factors in mine accidents. The model can predict the failure probability of human factors. Next, an evaluation index system for human reliability of mine operators was constructed. On this basis, fuzzy comprehensive evaluation (FCE) and analytic hierarchy process (AHP) were combined with neural network (NN) to evaluate human reliability of mine operators with stable performance. Meanwhile, principal component analysis (PCA) was combined with NN to evaluate human reliability of mine operators with unstable performance. Compared with the traditional evaluation methods, the proposed model reduces the dimension of input vector set, tolerates incomplete data samples, and achieves a high prediction accuracy.
\end{abstract}

\section{INTRODUCTION}

Mining has a much higher occurrence of serious and fatal accidents than other industries. Most mine accidents arise from unsafe behaviors of operators and unsafe state of objects. In recent years, the unsafe state of objects has been effectively eliminated, thanks to the great progress in mine safety management. The progress is the result of multiple factors: the modernization of mining equipment, the safety improvement of production system, and the advancement in various technologies (ventilation, safety monitoring, fire control, dust control, etc.). However, the progress on the control of unsafe behaviors is relatively slow.

Human behaviors are a direct cause of accidents, and an influencer of the unsafe state of objects. In general, 70\%-90\% of all accidents are directly or indirectly induced by human error; the ratio is even higher in the mining industry $[1,2]$. In the last three decades, $96.5 \%$ of mine accidents in China were resulted from human error [3]. Suffice it to say that human error is the leading hazard of mine safety. To reduce human error, it is necessary to accurately evaluate human reliability.

Despite drawing much attention, human reliability has not been evaluated with sufficient data or perfect models. There is not yet a report that quantifies human reliability in mine systems, which features heavy hazard sources, highly complex operations, harsh working conditions, various types of work, and low quality of operators. This calls for in-depth analysis on human factors in mine accidents, correct identification of unsafe behaviors of operators, and accurate evaluation of human reliability in mine system.

The mine is a complex system involving the interaction between operators, equipment, and environment. Besides equipment reliability and environmental impact, the human reliability of operators has a nonnegligible influence on mine safety. By evaluating the human reliability, it is possible to find the behavior patterns of operators and detect their psychological problems in time. Then, new countermeasures could be put forward to prevent and control mine accidents.

In this paper, a new correlation analysis model is created based on Apriori algorithm to predict the failure probability of human factors in mine accidents, and an evaluation index system was set up for reliability of mine operators. Next, the human reliabilities of mine operators with stable performance and with unstable performance were evaluated by fuzzy comprehensive evaluation (FCE) + analytic hierarchy process $(\mathrm{AHP})+$ neural network $(\mathrm{NN})$ and principal component analysis (PCA) + neutral network (NN), respectively. The superiority of our model was proved through example analysis.

\section{LITERATURE REVIEW}

Human reliability refers to the probability that operators complete a task within the specified time in any stage of system operation [4]. As an emerging subject, the evaluation of human reliability aims to quantify and qualify the reliability of operators, making it possible to analyze, predict and reduce human error [5]. As stated by Kirwan [6], human reliability evaluation mainly derives the probability of accidents induced by human error, and finds the way to minimize human error.

The development of human reliability evaluation can be divided into two stages. In the first stage, the evaluation models for human reliability are grounded on the theory of human behaviors. These models are static in nature, because they treat human behaviors like machine actions [7, 8]. In the second stage, the evaluation models focus on the dynamic cognitive process of human in specific scenarios, and tackle the mechanism of human error in the event of accidents. Moreover, the evaluation fully considers the operation experience of industrial system, as well as the measured or 
simulated information [9]. In recent years, the emphasis of human reliability evaluation shifts to the application of existing methods in various fields. To lower the possibility of human error, De Felice et al. [10] highlighted the importance of monitoring human reliability in railway transport, and evaluated the human reliability in railway operations with a knowledge-based system. Akyuz and Celik [11] integrated the AHP to the human error assessment and reduction technique (HEART), calculated the impact of human error by adjusting the weight ratio of human factors, analyzed the human reliability in chemical cleaning of oil tanks, enhancing the shipping reliability of hazardous chemicals. Kim and Kim [12] pointed out that traditional probabilistic safety analyses often view the probability of human-induced events as part of the probability of triggering events, and determined the humaninduced events that trigger low power consumption and shutdown of nuclear power plants. Considering the features of various analysis methods, Sun and Bin [13] examined the factors affecting human reliability in software testing, and proposed feasible solutions that enhance human reliability in software testing. Using Bayesian network, Liao et al. [14] quantified the probability of unsafe behaviors caused by design failure, and designed an economic and effective way to improve system safety: preventing accidents through design.

Many scholars have evaluated human reliability in mine operations. Xu et al. [15] conducted an empirical analysis with cognitive reliability and error analysis method (CREAM), put the failure probability of underground driving and drilling at 0.025 , and suggested enhancing human reliability in mine excavation by reducing the probability of the most possible failure mode of drilling. Wang et al. [16] proposed a method to predict and evaluate human error in mine operations, and applied the method to assess the reliability of operators in the mine. Drawing on grey correlation theory, Li et al. [17] quantified the CREAM control mode, and modelled the relationship between probability of human error (HEP) and degree of grey correlation. Wang et al. [16] established a human error risk analysis framework for emergency evacuation in mines, which includes scenario and task analysis, risk assessment, and risk reduction; human safety barriers were divided into organization, group and personal levels.

\section{MODEL CONSTRUCTION}

Quantitative evaluation of human reliability or HEP can find out the fundamental cause of human error. In this section, the CREAM method of human reliability analysis method is used to trace and analyze the occurred accidents, and to explore the unobservable human factors which caused the accidents. A new human reliability analysis model is established by combining the HEP prediction technology with the error analysis method by using Apriori algorithm.

\subsection{Selection of evaluation method}

Each human reliability evaluation method has its merits and defects. There is no evaluation method that applies to human reliability in all systems. In many systems, it is necessary to combined two or more evaluation methods. Technique for human error rate prediction (THERP) [18] decomposes each human task into several subtasks, sets up an event tree with the task as the trunk, assigns the failure probability of each subtasks on each branch by looking up the table, and computes the failure probability of the task by weighting those of all branches. This approach can accurately depict the operation features of every task, yet fails to reflect the effect of human cognition on accidents. To makes matters worse, the weighted calculation is easy to accumulate error, and the failure probability table of THERP is not suitable for all kinds of tasks.

CREAM [19] builds up an effective and reasonable failure probability model, which identifies the cognitive control mode of the cognitive behavior in each branch, assigns the failure probability to the identified cognitive control mode by looking up the table, and adjusts the probability based on the performance factors that reflect the features of situational environment (because the situation environment might affect human cognitive behavior). The failure probability evaluation model is well known for its reliability and accuracy in data quantification. As a result, the CREAM has been widely adopted to evaluate human reliability.

The combination of THERP and CREAM can give full play to their merits, and avoid their defects: the task decomposition and event tree construction are realized by THERP, while the failure probability is estimated in the light of the basic failure probability and the performance factors given by CREAM. In this way, the failure probability estimation will be more practical, and the human reliability evaluation will be more accurate.

The THERP-based quantification of failure probability consists of four stages: system familiarization, qualitative analysis, quantitative analysis, and result application. During qualitative analysis, the action types, action objects, and potential human errors of a task are analyzed in details, and the possible wrong actions and their types are identified. On this basis, the task is decomposed into a series of subtasks, and depicted with an event tree of human reliability evaluation.

After task decomposition, the subtasks have two logic relations: series and parallel. If the subtasks are connected in series, then task can succeed only if all subtasks are successful; if the subtasks are in parallel, then the task can succeed if any subtask is successfully. The success and failure probabilities of the task with series subtasks and the task with parallel subtasks can be quantified respectively by:

$$
\begin{gathered}
\left\{\begin{array}{c}
P(S)=u(v \mid u) \\
P(F)=1-u(v \mid u)=u(V \mid u)+U(v \mid U)+U(V \mid U)
\end{array}\right. \\
\left\{\begin{array}{c}
P(S)=U(V \mid U)=u(v \mid u)+u(V \mid u)+U(v \mid U) \\
P(F)=U(V \mid U)
\end{array}\right.
\end{gathered}
$$

where, $S$ and $F$ are the success and failure of the task, respectively; $U$ and $V$ are two subtasks; $u$ and $v$ are success and success probability of a subtask, respectively.

In the event tree of human reliability evaluation, HEP is affected by operator quality, current state of individuals, hardware reliability, management mechanism, and environmental conditions. The actual HEP of each subtask must be modified based on performance factors. Since the HEP is a conditional probability, it is necessary to consider the correlation between subtasks and operators in the system. Otherwise, the predicted HEP will be seriously distorted. Suppose a task can be logically divided into subtask $U$ and subtask $V$ in time order, that is, subtas $U$ occurs before subtask $V$. If subtask $U \mathrm{k}$ fails, then the failure probability of $V$ falls in one of the five levels according to the correlation between $U$ and $V$ :

Full correlation: 


$$
P(V \mid U)=1
$$

High correlation:

$$
P(V \mid U)=(1+P(V)) / 2
$$

Medium correlation:

$$
P(V \mid U)=(1+6 P(V)) / 7
$$

Low correlation:

$$
P(V \mid U)=(1+19 P(V)) / 20
$$

Zero correlation:

$$
P(V \mid U)=P(V)
$$

\subsection{Improved evaluation model}

CREAM holds that human error arises from incorrect cognition of the situation and wrong decision induced by the environment, in addition to wrong actions. There are four kinds of cognitive control modes in CREAM: scrambled, opportunistic, tactical and strategic [19]. The error probability interval of each mode is given in Table 1.

Table 1. Error probability interval of each mode

\begin{tabular}{cc}
\hline Cognitive control mode & Error probability interval \\
\hline Scrambled & $0.1<P<1.0$ \\
Opportunistic & $0.01<P<0.5$ \\
Tactical & $0.001<P<0.1$ \\
Strategic & $0.00005<P<0.01$ \\
\hline
\end{tabular}

CREAM classifies the factors affecting cognitive control mode and cognition effect into nine categories, which are collectively referred to as common performance conditions (CPCs). Each factor is called a CPC factor. The correlation between CPC factors can be analyzed by the Apriori algorithm [20], a common mining technique for data association rules.

Apriori algorithm looks for the frequent item sets in the target dataset by two metrics, namely, support and confidence. Support is the number of occurrences of each member item. The support of the pair of frequent items $X$ and $Y$ can be defined as:

$$
\operatorname{Support}(\mathrm{X}, \mathrm{Y})=\mathrm{P}(\mathrm{XY})=\operatorname{Num}(X Y) / N u m(\text { Allsamples })
$$

In general, an item with a high support or a too low support does not belong to the frequent item set.

Confidence is the probability that if an item occurs, then another item will also occur. The confidence of $X$ on $Y$ can be defined as:

$$
\text { Confidence }(\mathrm{X} \Leftarrow \mathrm{Y})=\mathrm{P}(\mathrm{X} \mid \mathrm{Y})=P(X Y) / P(Y)
$$

Here, Apriori algorithm is introduced to analyze the correlation between human factors. Then, the weighting of cognitive control mode in CREAM was modified based on the historical data on human-induced mine accidents. Based on the mined association rules, six factors were found to have great impact on the occurrence of cognitive error: underground monitoring system, underground personnel positioning system, underground emergency shelter system, underground compressed air self-rescue system, underground water supply rescue system, and underground communication system. Therefore, the six factors were taken as the CPC factors in the mine system.

According to its degree of impact on operator performance, each CPC factor was divided into three levels: improved, reduced and insignificant. Then, the probability of cognitive error leading to human-induced mine accidents was predicted by an improved method: the probability of cognitive failure was calculated based on the $\sum_{\text {reduced }}$ and $\sum_{\text {improved }}$ of CPC factors:

$$
\eta=\Sigma_{\text {reduced }}-\Sigma_{\text {improved }}
$$

where, $\eta$ is the context influence index; $\sum_{\text {reduced }}$ and $\sum_{\text {improved }}$ are the number of CPC factors whose level is reduced or improved, respectively.

If there is no context impact, the context influence index $\eta$ is zero. At this time, the probability of cognitive failure is the basic failure probability $C F P_{0}$. The relationship between cognitive failure probability $C F P$, basic failure probability $C F P_{0}$ and context influence index $\eta$ can be expressed as:

$$
\lg \left(C F P / C F P_{0}\right)=k \eta
$$

The basic failure probability $C F P_{0}$ depends on $C F P$ and $\eta_{\max }$, and $\eta_{\min }$ :

$$
\begin{aligned}
& \eta_{\max }=\lg \left(C F P_{\max } / C F P_{0}\right) / k \\
& \eta_{\min }=\lg \left(C F P_{\min } / C F P_{0}\right) / k
\end{aligned}
$$

Thus, $k$ and $C F P_{0}$ can be respectively expressed as:

$$
\begin{gathered}
k=\lg \left(C F P_{\text {max }} / C F P_{\text {min }}\right)=\left(\eta_{\max }-\eta_{\min }\right) \\
C F P_{0}=C F P_{\text {max }} / 10^{k \eta_{\text {max }}}
\end{gathered}
$$

Then, the cognitive failure probability $C F P$ can be calculated by:

$$
\mathrm{CFP}=C F P_{0} \times 10^{\frac{\eta}{4}}
$$

The improved evaluation model for human factors in mine accidents involves the following steps:

Step 1. For the subtasks on each branch of the event tree, analyze the cognitive activities in human-induced accident, and determine the cognitive control mode of these activities.

Step 2. Find the basic cognitive failure probability $C F P_{0}$.

Step 3. Under the context of cognitive activities, determine the level of each CPC factor, quantify the effect of each factor, and adjust the weight of the cognitive control mode, using Apriori algorithm.

Step 4. Compute the context influence index, and calculate the cognitive failure probability of each subtask by (16).

Step 5. According to the logic relation of subtasks in event tree, weight or multiply the cognitive failure probabilities of the member subtasks to obtain the cognitive failure probability of each branch.

Table 2 lists the type of cognitive function, cognitive control mode, and basic failure probability of each branch in the event tree, which are obtained by our model. 
Table 2. Cognitive function type, cognitive control mode and basic failure probability of each branch

\begin{tabular}{ccccc}
\hline Code & Human error & $\begin{array}{c}\text { Cognitive } \\
\text { function }\end{array}$ & $\begin{array}{c}\text { Cognitive control } \\
\text { mode }\end{array}$ & $\begin{array}{c}\text { Basic failure } \\
\text { probability }\end{array}$ \\
\hline $\mathrm{P}_{1}$ & The operator manually turns off the sensor. & Planning & Inappropriate plan & 0.02 \\
$\mathrm{P}_{2}$ & The alarm is ignored. & Observation & Failure to respond to observation & 0.008 \\
$\mathrm{P}_{3}$ & The hazard is not noticed by monitoring personnel. & Observation & Failure to respond to observation & 0.008 \\
$\mathrm{P}_{4}$ & The instruction is incorrect. & Explanation & Wrong decision & 0.01 \\
$\mathrm{P}_{5}$ & The instruction is not executed correctly. & Execution & Wrong action & 0.03 \\
$\mathrm{P}_{6}$ & The incorrect instruction is executed. & Execution & Wrong target & 0.005 \\
\hline
\end{tabular}

\section{HUMAN RELIABILITY EVALUATION}

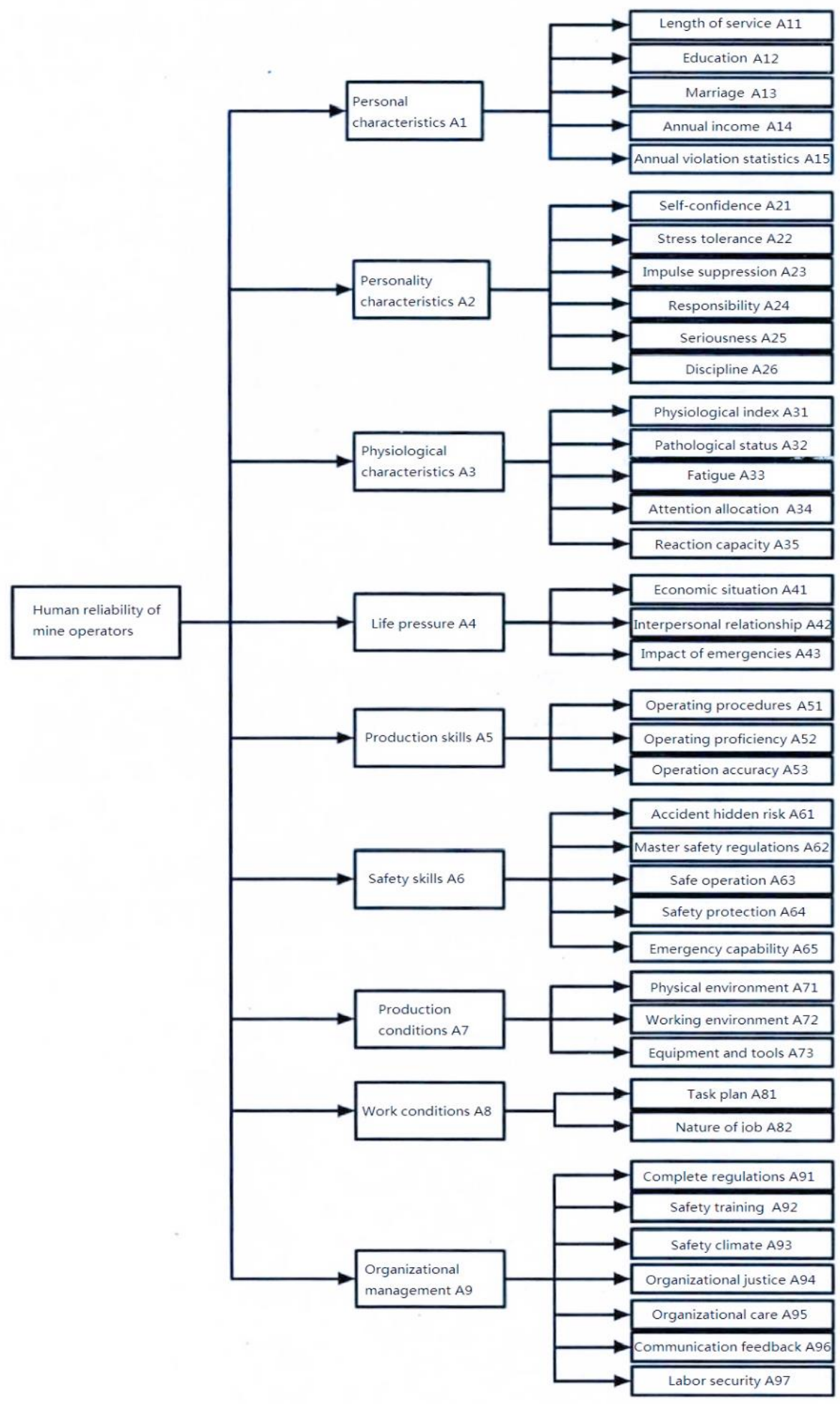

Figure 1. Evaluation index system of human reliability for mine operators 


\subsection{Evaluation index system}

Many studies [21, 22] have shown that the safety management of mines and unsafe behaviors of operators are greatly affected by internal factors like personality and physiology and external factors like work conditions, production conditions, and organizational management. Based on abundant survey data and relevant findings, this paper sets up an evaluation index system of human reliability for mine operators.

As shown in Figure 1 above, the evaluation index system contains nine indices, namely, personal characteristics, personality characteristics, physiological characteristics, life pressure, production skills, safety skills, production conditions, work conditions, and organizational management. Each index was decomposed into a number of sub-indices.

The index weight reflects the importance of an index to the object of evaluation. To weight the indices reasonably, the AHP was introduced to determine the fuzzy weight vector of each index in the FCE model. This is because the AHP is good at solving complex decision-making problems with multiple influencing factors.

The weighted average operator was adopted in the FCE model. Firstly, the sub-indices were synthetized by fuzzy method. Then, the calculated results were combined into the FCE matrix of the factor layer. Finally, the weight vectors and FCE matrix were integrated into the result vector of the FCE.

The above method mainly aims at operators with stable performance. These operators have relatively complete file data and safety data, making it easy to extract the index data. It is suitable to implement FCE to evaluate the human factors of such operators. However, there are also operators with unstable performance, due to the limited experience. Another evaluation method is designed for them in the following subsection.

\subsection{NN-based multi-index comprehensive evaluation}

Considering the advantages of $\mathrm{NN}$ in self-learning, adaptability and fault tolerance, an NN-based comprehensive evaluation model was established, mimicking the human thinking pattern. The model combines qualitative and quantitative evaluations, eliminating the subjectivity and uncertainty of manually assigned weights and correlation coefficients. The input parameters were obtained by analyzing various factors affecting human reliability. Then, the NN was applied to evaluate the human reliability of mine operators with unstable performance.

The NN of our model was extended from radial basis function (RBF) NN, a feedforward $\mathrm{NN}$ capable of global search and nonlinear learning. Featuring simple structure and fast convergence, the RBFNN has been widely used in pattern recognition, information classification, and function approximation. Compared with the backpropagation neural network (BPNN), the RBFNN boasts an efficient iterative process and avoids the local optimum trap by adopting the local activation function.

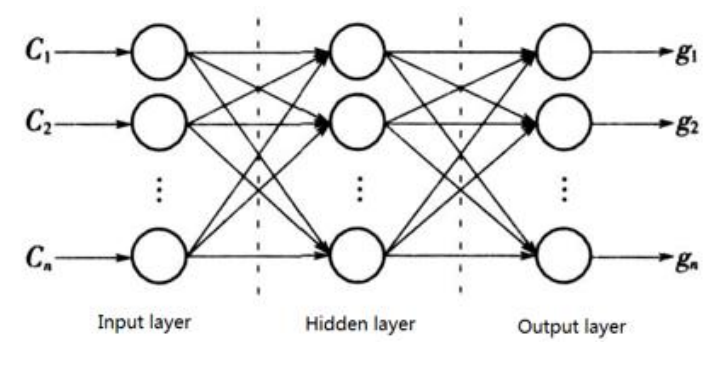

Figure 2. Structure of the RBFNN

As shown in Figure 2, the RBFNN has many more neurons in the hidden layer than the standard BPNN. The hidden layer mainly responds to the input signal. More neurons in this layer mean a larger output from the center of the basis function. Therefore, the RBFNN enjoys good local approximation ability.

The structural complexity and prediction accuracy of RBFNN directly hinge on the number of input variables. If the input variables are too many and strongly corelated, the RBFNN will have a complex structure, consume lots of time in training, and face large errors in prediction. To mitigate these risks, the RBFNN was coupled with the PCA to reduce the dimension of evaluation indices.

In the PCA-RBFNN model, the original variable set was modelled by the PCA to eliminate the correlation between original variables. Then, the variables whose cumulative contribution is above $85 \%$ were treated as the new input sample of RBFNN simulation. Prior to the PCA, the raw data were normalized, including but not limited to quantification of qualitative indies, forward processing of reverse indices, removal of extremums [23].

As for the RBFNN parameters, the center and variance of hidden layer basis function were learned through selforganization, while the weight between the hidden layer and the output layer was determined through supervised learning.

\subsection{Example of human reliability prediction}

A total of 20 mine operators were trained as the subjects. Two test courses were randomly selected from 19 common working procedures of driving posts. The evaluation of human reliability aims to measure the post adaptability and safety of these operators. By the nature of task, human reliability falls into operational reliability, monitoring reliability, and management reliability. The human reliability of operators engaging in driving operation is mainly operational reliability. Table 3 provides the scale of human reliability evaluation.

The human reliability in the example is influenced by 143 factors in four categories: human factors, equipment and operation factors, management factors, and environmental factors. Many indices in terms of physiology, safety management, and family information are not available. The data on equipment and operation factors are relatively complete. Therefore, these factors were normalized as Table 4 before going through the PCA.

Table 3. Human reliability scale

\begin{tabular}{cccccc}
\hline Unsafe behavior & $\mathbf{5 5}$ & $\mathbf{6 - 9}$ & $\mathbf{1 0 - 1 2}$ & $\mathbf{1 3 - 1 5}$ & $\geq \mathbf{1 6}$ \\
\hline Reliability levels & Strongly reliable & Slightly reliable & Neutral & Slightly unreliable & Strongly unreliable \\
Post test results & Excellent & Good & Qualified & Retraining & Laid off \\
Network output & 10000 & 01000 & 00100 & 00010 & 00001 \\
Initial value of reliability & 0.85 & 0.70 & 0.60 & 0.50 & 0.40 \\
\hline
\end{tabular}


Table 4. Normalization of equipment and operation factors

\begin{tabular}{cccccc}
\hline & $\mathbf{A}_{\mathbf{1 1}}$ & $\mathbf{A}_{\mathbf{1 2}}$ & $\mathbf{A}_{\mathbf{1 3}}$ & $\mathbf{A}_{\mathbf{1 4}}$ & $\mathbf{A}_{\mathbf{1 5}}$ \\
\hline W1 & -0.9752 & -0.8705 & -0.8219 & 0.6728 & 0.1844 \\
W2 & -0.9752 & -0.8705 & -1.4290 & 0.6728 & 1.3665 \\
W3 & -0.9752 & 0.0529 & -2.5174 & -1.2764 & 1.3665 \\
W4 & 0.2276 & 0.0529 & 0.4194 & -1.2764 & -0.4098 \\
W5 & 0.2276 & 0.0529 & 0.4194 & 1.3856 & 1.3665 \\
W6 & 0.2276 & 0.0529 & 0.4194 & -0.2951 & 0.1844 \\
W7 & -3.1876 & -2.1372 & -2.5174 & -1.2764 & 0.1844 \\
W8 & 0.2276 & 0.0529 & 0.4194 & 0.6728 & 0.1844 \\
W9 & 0.2276 & 0.0529 & 0.4194 & 0.6728 & -2.3817 \\
W10 & 0.2276 & 0.0529 & 1.2258 & 0.6728 & 0.1844 \\
W11 & 0.2276 & -0.8705 & -0.5328 & -0.2951 & 0.1844 \\
W12 & 1.1739 & 0.0529 & 0.4194 & 0.6728 & 0.1844 \\
W13 & 0.2276 & 0.0529 & 0.4194 & 0.6728 & -0.4098 \\
W14 & 0.2276 & 1.2833 & 0.4194 & 1.3856 & 0.1844 \\
W15 & 0.2276 & -0.8705 & 0.4194 & -1.2764 & 1.3665 \\
W16 & -0.9752 & 1.2833 & -1.4290 & -1.2764 & 1.3665 \\
W17 & 0.2276 & 1.2833 & 0.4194 & -0.2951 & 0.1844 \\
W18 & 1.1739 & 0.0529 & 0.4194 & 1.3856 & 0.1844 \\
W19 & 0.2276 & 0.0529 & 0.4194 & 0.6728 & -0.4098 \\
W20 & 0.2276 & -0.8705 & 1.2258 & -0.2951 & 1.3665 \\
\hline
\end{tabular}

Taking the normalized values as inputs, the correlation matrix was obtained, and then the common factor variance was calculated, reflecting the proportion of information extracted from each index in our evaluation index system. Finally, the total variance explained was derived (Table 5).

Table 5. Variance explained

\begin{tabular}{ccccc}
\hline $\begin{array}{c}\text { Unsafe } \\
\text { behavior }\end{array}$ & \multicolumn{4}{c}{$\begin{array}{c}\text { Initial } \\
\text { eigenvalue }\end{array}$} \\
\hline & Total & Variance & Cumulative & Eigenvalue \\
1 & 3.163 & 42.87 & 42.87 & 1.782 \\
2 & 2.115 & 25.31 & 69.18 & 1.892 \\
3 & 1.227 & 15.93 & 84.11 & 1.272 \\
4 & 0.385 & 4.82 & 88.93 & 0.952 \\
5 & 0.276 & 4.19 & 93.12 & 0.738 \\
6 & 0.257 & 3.08 & 96.20 & 0.731 \\
7 & 0.187 & 2.59 & 98.79 & 0.598 \\
8 & 0.083 & 1.21 & 100 & 0.483 \\
\hline
\end{tabular}

As shown in Table 5, three eigenvalues were greater than 1 , whose cumulative variance reached $84.11 \%$. The three principal components can restore $84.11 \%$ of the original information, surpassing the required level of $80 \%$.

Then, the normalized data on these principal components were obtained by the PCA, and inputted to the RBFNN. From 20 groups of effective samples, 16 groups were randomly selected as training samples, and the remaining 4 as test samples. The RBFNN outputs for the four test samples are listed in Table 6.

Table 6. Human reliability ratings

\begin{tabular}{cccc}
\hline Sample & Expected output & Actual output & Reliability \\
\hline $\boldsymbol{W} 1^{\prime}$ & 00100 & 00100 & Neutral \\
$\boldsymbol{W} 2^{\prime}$ & 01000 & 01000 & Slightly reliable \\
$\boldsymbol{W} 3^{\prime}$ & 10000 & 10000 & Strongly reliable \\
$\boldsymbol{W} 4^{\prime}$ & 00100 & 00100 & Neutral \\
\hline
\end{tabular}

As shown in Table 6, the output vectors of the test samples agreed well with the expected outputs, and conformed to the result of human reliability evaluation. Moreover, the initial value of reliability was given reasonable credibility.

\section{CONCLUSIONS}

Human factors play an important role in mine accidents. It is an urgent need to evaluate the human reliability of mine operators and reduce human-induced accidents. This paper fully integrates the ideas and methods of multiple disciplines (e.g. system engineering, safety engineering, and safety psychology) to analyze and evaluate human reliability in mine operation, and obtains some exploratory results.

Specifically, a novel correlation analysis model was established to predict the failure probability of human factors in mine system, which is grounded on Apriori algorithm, THERP, and CREAM. On this basis, an evaluation index system was established for human reliability of mine operators. Next, the human reliabilities of mine operators with stable performance and with unstable performance were evaluated by $\mathrm{FCE}+\mathrm{AHP}+\mathrm{NN}$ and $\mathrm{PCA}+\mathrm{NN}$, respectively.

During the evaluation, the input vector set of RBFNN was reduced through the PCA, and the principal components were obtained based on the contribution rate, creating the original sample space. In this way, the dimensionality of the input vector set was reduced, and the correlations between input vectors were eliminated, without sacrificing the main information of the original vector set. Hence, the evaluation problem was simplified, while the RBFNN became faster in evaluation, and acquired stronger generalization ability.

\section{REFERENCES}

[1] Rukšènas, R., Curzon, P., Blandford, A., Back, J. (2014). Combining human error verification and timing analysis: a case study on an infusion pump. Formal Aspects of Computing, 26(5): https://doi.org/10.1007/s00165-013-0288-1

[2] Anu, V., Hu, W., Carver, J.C., Walia, G.S., Bradshaw, G. (2018). Development of a human error taxonomy for software requirements: A systematic literature review. Information and Software Technology, 103: 112-124. https://doi.org/10.1016/j.infsof.2018.06.011

[3] Liu, R., Cheng, W., Yu, Y., Xu, Q. (2018). Human factors analysis of major coal mine accidents in China based on the HFACS-CM model and AHP method. International Journal of Industrial Ergonomics, 68: 270279. https://doi.org/10.1016/j.ergon.2018.08.009

[4] Dhillon, B.S. (2013). Human Reliability: With Human Factors. Elsevier.

[5] Martorell, P., Martón, I., Sánchez, A.I., Martorell, S., Sanchez-Saez, F., Saiz, M. (2018). Evaluation of risk impact of completion time changes combining PSA and DSA model insight and human reliability analysis. Reliability Engineering \& System Safety, 178: 97-107. https://doi.org/10.1016/j.ress.2018.05.008

[6] Kirwan, B. (1992). Human error identification in human reliability assessment. Part 2: Detailed comparison of techniques. Applied Ergonomics, 23(6): 371-381. https://doi.org/10.1016/0003-6870(92)90368-6

[7] Mosleh, A., Chang, Y.H. (2004). Model-based human reliability analysis: Prospects and requirements. Reliability Engineering \& System Safety, 83(2): 241-253. https://doi.org/10.1016/j.ress.2003.09.014

[8] Abdellaoui, M., Douik, A. (2020). Human action recognition in video sequences using deep belief networks. Traitement du Signal, 37(1): 37-44. 


\section{https://doi.org/10.18280/ts.370105}

[9] Podofillini, L., Dang, V.N. (2013). A Bayesian approach to treat expert-elicited probabilities in human reliability analysis model construction. Reliability Engineering \& System Safety, 117: 52-64. https://doi.org/10.1016/j.ress.2013.03.015

[10] De Felice, F., Petrillo, A. (2011). Methodological approach for performing human reliability and error analysis in railway transportation system. International Journal of Engineering and Technology, 3(5): 341-353.

[11] Akyuz, E., Celik, M. (2015). A methodological extension to human reliability analysis for cargo tank cleaning operation on board chemical tanker ships. Safety Science, 75: 146-155. https://doi.org/10.1016/j.ssci.2015.02.008

[12] Kim, Y., Kim, J. (2015). Identification of human-induced initiating events in the low power and shutdown operation using the Commission Error Search and Assessment method. Nuclear Engineering and Technology, 47(2): 187-195. https://doi.org/10.1016/j.net.2014.12.006

[13] Sun, G., Bin, S. (2018). A new opinion leaders detecting algorithm in multi-relationship online social networks. Multimedia Tools and Applications, 77(4): 4295-4307. https://doi.org/10.1007/s11042-017-4766-y

[14] Liao, P.C., Luo, X., Wang, T., Su, Y. (2016). The mechanism of how design failures cause unsafe behavior: the cognitive reliability and error analysis method (CREAM). Procedia Engineering, 145(6): 715-722. https://doi.org/10.1016/j.proeng.2016.04.088

[15] Xu, Y., Wu, K., Li, L., Zhou, D., Hu, Z. (2019). Ground cracks development and characteristics of strata movement under fast excavation: a case study at Bulianta coal mine, China. Bulletin of Engineering Geology and the Environment, 78(1): 325-340. https://doi.org/10.1007/s10064-017-1047-y

[16] Wang, L., Wang, Y., Cao, Q., Li, X., Li, J., Wu, X. (2014). A framework for human error risk analysis of coal mine emergency evacuation in China. Journal of
Loss Prevention in the Process Industries, 30: 113-123. https://doi.org/10.1016/j.jlp.2014.05.007

[17] Li, H., Chen, D., Arzaghi, E., Abbassi, R., Xu, B., Patelli, E., Tolo, S. (2018). Safety assessment of hydrogenerating units using experiments and grey-entropy correlation analysis. Energy, 165: 222-234. https://doi.org/10.1016/j.energy.2018.09.079

[18] Shirley, R.B., Smidts, C., Li, M., Gupta, A. (2015). Validating THERP: Assessing the scope of a full-scale validation of the Technique for Human Error Rate Prediction. Annals of Nuclear Energy, 77: 194-211. https://doi.org/10.1016/j.anucene.2014.10.017

[19] Friesen, B.V. (1963). Experiences with Delfen Cream as contraceptive method. Acta Obstetricia et Gynecologica Scandinavica, 42(s6): 71-73. https://doi.org/10.3109/00016346309158253

[20] Borghini, F., Garzia, F., Lombardi, M., Mete, M., Perruzza, R., Tartaglia, R. (2018). Human factor analysis inside a peculiar job environment at the Gran Sasso mountain underground laboratory of Italian National Institute for Nuclear Physics. International Journal of Safety and Security Engineering, 8(3): 390-405. https://doi.org/10.2495/SAFE-V8-N3-390-405

[21] Lin, C.J., Yenn, T.C., Jou, Y.T., Hsieh, T.L., Yang, C.W. (2013). Analyzing the staffing and workload in the main control room of the advanced nuclear power plant from the human information processing perspective. Safety Science, $\quad 57:$ 161-168. https://doi.org/10.1016/j.ssci.2013.02.004

[22] Kosmowski, K.T. (2011). Functional Safety Analysis including Human Factors. International Journal of Performability Engineering, 7(1): 61-76.

[23] Wang, L., Wang, S.G., Wu, D.L., Liu, H.H., Wang, J. (2019). An evaluation method for harmonic emission level based on principal component regression. European Journal of Electrical Engineering, 21(5): 415-420. https://doi.org/10.18280/ejee.210503 